\title{
Disruption of the ATE1 and SLC12A1 Genes by Balanced Translocation in a Boy with Non-Syndromic Hearing Loss
}

\author{
B. Vona ${ }^{a}$ C. Neuner ${ }^{a} \quad$ N. El Hajj ${ }^{a} \quad$ E. Schneider ${ }^{a}$ R. Farcas ${ }^{b}$ V. Beyer ${ }^{b}$

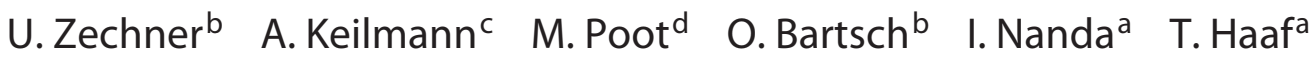 \\ a Institute of Human Genetics, Julius Maximilians University, Wuerzburg, ${ }^{b}$ Institute of Human Genetics, and \\ 'Division of Communication Disorders, Department of ORL, University Medical Center, Mainz, Germany; \\ ${ }^{\mathrm{d}}$ Department of Medical Genetics, University Medical Center, Utrecht, The Netherlands
}

\section{Key Words}

ATE1 - Disease-associated balanced chromosome rearrangement - Non-syndromic hearing impairment .

Reciprocal translocation · SLC12A1

\begin{abstract}
We report on a boy with non-syndromic hearing loss and an apparently balanced translocation $\mathrm{t}(10 ; 15)(\mathrm{q} 26.13 ; \mathrm{q} 21.1)$. The same translocation was found in the normally hearing brother, father and paternal grandfather; however, this does not exclude its involvement in disease pathogenesis, for example, by unmasking a second mutation. Breakpoint analysis via FISH with BAC clones and long-range PCR products revealed a disruption of the arginyltransferase 1 (ATE1) gene on translocation chromosome 10 and the solute carrier family 12, member 1 gene (SLC12A1) on translocation chromosome 15. SNP array analysis revealed neither loss nor gain of chromosomal regions in the affected child, and a targeted gene enrichment panel consisting of 130 known deafness genes was negative for pathogenic mutations. The expression patterns in zebrafish and humans did not provide evidence for ear-specific functions of the ATE 1 and SLC12A1 genes. Sanger sequencing of the 2 genes in the boy and 180 GJB2 mutation-negative hearing-impaired individuals did
\end{abstract}

\begin{tabular}{ll}
\hline KARGER & $\begin{array}{l}\text { ( } 2013 \text { S. Karger AG, Basel } \\
1661-8769 / 13 / 0051-0003 \$ 38.00 / 0\end{array}$ \\
$\begin{array}{l}\text { E-Mail karger@karger.com } \\
\text { www.karger.com/msy }\end{array}$ & $\begin{array}{l}\text { This is an Open Access article licensed under the terms of the } \\
\text { Creative Commons Attribution-NonCommercial } 3.0 \text { Un- } \\
\text { ported license (CC BY-NC) (www.karger.com/OA-license), } \\
\text { applicable to the online version of the article only. Distribu- } \\
\text { tion permitted for non-commercial purposes only. }\end{array}$
\end{tabular}

not detect homozygous or compound heterozygous pathogenic mutations. Our study demonstrates the many difficulties in unraveling the molecular causes of a heterogeneous phenotype. We cannot directly implicate disruption of ATE1 and/or SLC12A1 to the abnormal hearing phenotype; however, mutations in these genes may have a role in polygenic or multifactorial forms of hearing impairment. On the other hand, it is conceivable that our patient carries a diseasecausing mutation in a so far unidentified deafness gene. Evidently, disruption of ATE1 and/or SLC12A1 gene function alone does not have adverse effects. $\quad$ ○ $2013 \mathrm{~S}$. Karger AG, Basel

Autosomal reciprocal translocations affect approximately $0.1 \%$ of newborns [Hook and Hamerton, 1977]. When associated with abnormal phenotypes and/or developmental delay, apparently balanced chromosome rearrangements pose challenging situations in genetic counseling. Gene disruption by translocation or microdeletions/duplications in the breakpoint region or elsewhere in the genome may have phenotypic consequences. The latter are more frequent in de novo translocations with syndromic phenotypes, whereas gene disruption appears to occur at comparable frequencies in phenotypi- 
cally normal and abnormal translocation carriers [Baptista et al., 2008]. This argues in favor of the notion that in many cases gene disruption can be compensated for and has only mild or no phenotypic expression. It has been suggested that the breakpoints in non-syndromic patients may be associated with common and complex diseases [Bache et al., 2006]. Chromosomal rearrangements can cause haploinsufficiency not only by direct gene disruption, but also by position effects separating the coding region of a gene from regulatory elements occurring at a considerable distance as great as one megabase. This event may consequently lead to gene dysregulation [Kleinjan and van Heyningen, 2005]. Disease-associated balanced chromosome rearrangements provide a powerful tool for the identification of disease-causing genes [Bugge et al., 2000; Higgins et al., 2008].

If a balanced rearrangement is detected both in the carrier parent and offspring, then the risk for phenotypic abnormality is generally thought to be very low. Nonetheless, there are several studies reporting patients with abnormal phenotypes despite having the same balanced rearrangement as their phenotypically normal carrier parent [Warburton, 1991]. One possible explanation for discordant phenotypes in carriers of the same familial translocation or inversion is segregation of a second genetic mutation on the homologous chromosomes (compound heterozygosity) or elsewhere in the genome (digenic/polygenic inheritance). Furthermore, additional cryptic genomic imbalances may arise in the germline of balanced translocation carriers due to non-homologous recombination events.

We identified a family with a balanced reciprocal translocation $\mathrm{t}(10 ; 15)(\mathrm{q} 26.13 ; \mathrm{q} 21.1)$ that was inherited through 3 generations. All translocation carriers were healthy with no reported abnormalities except non-syndromic sensorineural hearing loss affecting one child. Hearing impairment is estimated to affect one out of every 1,000 newborns and increases to 3.5 out of 1,000 individuals by 18 years of age [Morton and Nance, 2006]. Furthermore, it is hypothesized that up to $1 \%$ of the greater than 20,000 genes in humans are necessary for hearing [Friedman and Griffith, 2003]. If roughly 200 genes are anticipated to play various roles in hearing and given that the number of known deafness genes (http://hereditaryhearingloss.org) are fewer than the suspected number, then there are many more deafness genes remaining to be discovered. We analyzed the chromosome breakpoint regions of our proband with the idea to identify a candidate gene(s) for hearing impairment. Previous studies have already demonstrated that in a given patient/family, dis- ease-associated balanced chromosome rearrangements can provide a visible bridge between hearing impairment and the underlying genotype [Williamson et al., 2007; Damatova et al., 2009; Schneider et al., 2009].

\section{Material and Methods}

Classic and Molecular Cytogenetic Analyses

Cytogenetic analyses were performed on the index patient as well as his parents, paternal grandparents and brother from peripheral blood lymphocyte cultures using conventional GTGbanding techniques at the 550-band level.

To delineate the breakpoint regions, FISH was carried out using selected BAC probes from the chromosomal 10q25.3q26.13 and $15 \mathrm{q} 15 \mathrm{q} 21.3$ regions (online suppl. table 1; for all online suppl. material, see www.karger.com/doi/10.1159/000355443). The locations of these BAC clones were chosen from the Ensembl browser (GRCh37) and ordered from the Children's Hospital Oakland Research Institute (Oakland, Calif., USA). In order to amplify larger BAC subfragments ranging from 3.5 to $10.7 \mathrm{~kb}$, the Expand Long Template PCR System (Roche Diagnostics, Mannheim, Germany) was used according to the manufacturer's recommendations with a series of primer pairs (online suppl. table 2) chosen from the genomic sequence of the breakpoint clones.

Detection of CNVs was performed with an Illumina CytoSNP-12 v2 microarray (Illumina Inc., San Diego, Calif., USA) according to the manufacturer's protocol. Array data were analyzed using GenomeStudio version 2011.1 and cnvPartition 3.2.0 (Illumina). A minimum cut-off value of 5 probes with a consistently aberrant signal was included in our criteria to ascertain a copy number change.

Sanger Sequencing and Targeted Next Generation Sequencing of Deafness Genes

Genomic DNA was extracted from whole blood using a standard salt extraction method. PCR was performed with an initial denaturation at $95^{\circ} \mathrm{C}$ for $3 \mathrm{~min}, 25$ cycles of $95^{\circ} \mathrm{C}$ for $30 \mathrm{~s}, 60^{\circ} \mathrm{C}$ for $30 \mathrm{~s}, 72^{\circ} \mathrm{C}$ for $30 \mathrm{~s}$, and a final $10 \mathrm{~min}$ extension using M13 primers (Metabion, Munich, Germany) (online suppl. table 3), PCR buffer, nucleotide mixture and Fast Start Taq Polymerase (Roche Diagnostics). The 12 exons of ATE1 and 26 coding exons of SLC12A1 were Sanger sequenced with an ABI PRISM 377 (Applied Biosystems, Life Technologies, Carlsbad, Calif., USA). The sequence reaction was completed with $5 \times$ sequencing buffer and big dye terminator (Applied Biosystems). DNA sequence analysis was performed using NCBI BLAST. In the case of non-synonymous substitutions, PolyPhen-2 [Adzhubei et al., 2010], SIFT [Ng and Henikoff, 2001] and Alamut (Interactive Biosoftware, Rouen, France) were used to predict the impact of any identified amino acid substitution on the protein structure and function and the disease-causing potential.

Genomic DNA of the proband was submitted to Otogenetics Corporation (Norcross, Ga., USA) for exome capture targeting 130 known deafness genes and sequencing on a HiSeq2000 (Illumina). Paired-end reads of $90-100 \mathrm{bp}$ were analyzed for quality, exome coverage and exome-wide SNP/InDels using the platform provided by DNAnexus (Mountain View, Calif., USA).
$4 \quad$ Mol Syndromol 2014;5:3-10 DOI: $10.1159 / 000355443$
Vona et al. 


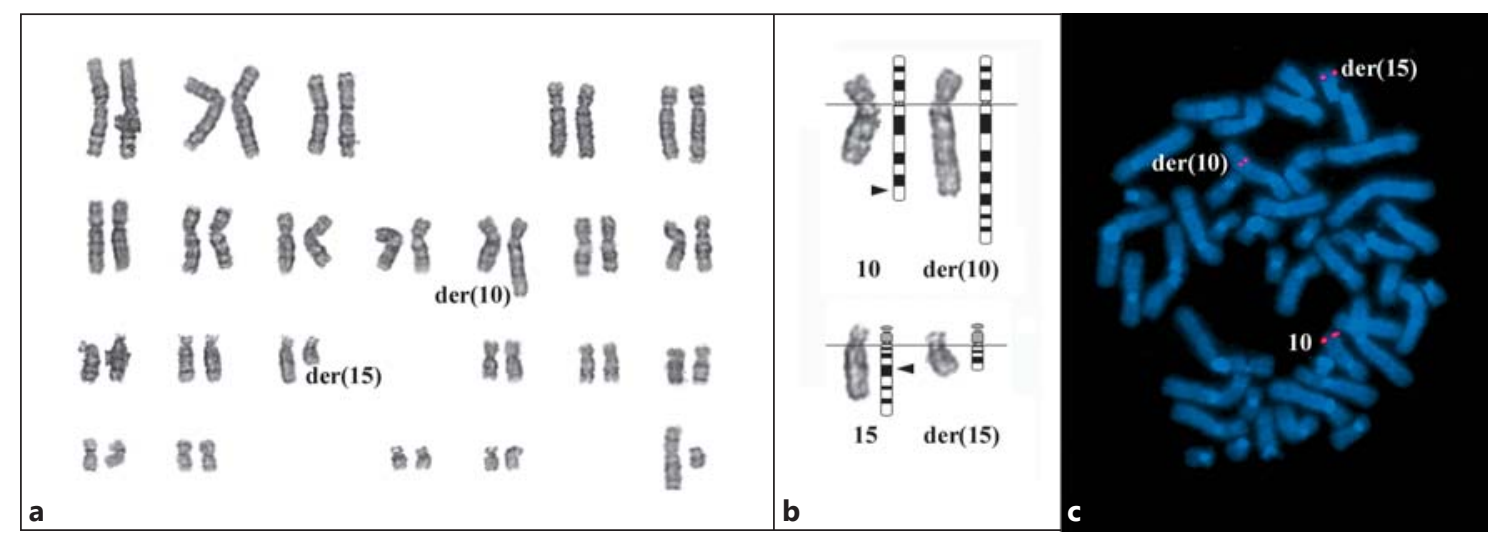

Fig. 1. Chromosome banding and FISH analysis of breakpoint regions. a G-banded karyotype of the index patient showing the apparently balanced derivative chromosomes. b G-banding pattern as well as corresponding ideograms showing the normal and derivative chromosomes resulting from the $\mathrm{t}(10 ; 15)(\mathrm{q} 26.13 ; \mathrm{q} 21.1)$ translocation. Arrowheads indicate the translocation breakpoints. c DAPI-stained (blue) metaphase spread of the index patient hybridized with digoxigenin-labeled (red) BAC RP11-78A18. The breakpoint-spanning BAC highlights the ATE1 gene on the normal chromosome 10 as well as the 2 derivative chromosomes 10 and 15.

\section{Zebrafish Whole Mount in situ Hybridization}

Whole mount in situ hybridization was done to assess ate 1 and slc12al expression in wild-type developing zebrafish (Danio rerio, wild-type $\mathrm{TüAB}$ strain) embryos. Antisense riboprobes for ate1 and slc12a1 were prepared using the pCRII-TOPO vector (Invitrogen, Darmstadt, Germany). A 646-bp fragment of ate1 on zebrafish chr.Zv9_NA48 was isolated from the cDNA of whole embryos (26-28 hours post fertilization, hpf) using forward primer $5^{\prime}$-GGCCTCCTCCTCAAGTCTCT- $3^{\prime}$ and reverse primer $5^{\prime}$-GACACACAAGAGGGCAGGAT- $3^{\prime}$ and was cloned into a pCRIITOPO vector. For preparation of the ate 1 antisense riboprobe, pCRII-ate1 was digested with BamHI and transcribed with T7 RNA polymerase. Similarly, a 505-bp fragment of slc12a1 on zebrafish chromosome 18 was isolated using forward primer $5^{\prime}$-TCTGCCTAAAGGGACTCTGC- $3^{\prime}$ and reverse primer $5^{\prime}$-CGAGGCCAGAAAGAAGTTGG-3'. The fragment was cloned into a pCRII-TOPO vector. For preparation of the slc12a1 antisense riboprobe, pCRII-slc12al was digested with HindIII and transcribed with T7 RNA polymerase. In situ hybridization was performed as described in Winkler and Moon [2001]. Embryos were mounted in glycerol and photographed as whole mounts.

\section{Results}

\section{Clinical Report}

The patient is the first child of non-consanguineous German parents without a family history of hearing loss in childhood. His grandparents, parents and younger brother were healthy and normally hearing. Following an uneventful pregnancy, he was born at term with Apgar scores of 10 and 10 at 1 and $5 \mathrm{~min}$, respectively. Birth weight was 5,300 $\mathrm{g}(+4 \mathrm{SD})$, length was $62 \mathrm{~cm}(+4.5 \mathrm{SD})$ and occipitofrontal circumference $39 \mathrm{~cm}(+3.7 \mathrm{SD})$. The reasons for the macrosomia and macrocephaly remained unknown; in particular, there was no evidence for an overgrowth syndrome or for gestational diabetes. Newborn hearing screening (otoacoustic emission) was not performed. Development was normal and without notable diseases, and his overgrowth gradually decreased to normal. He could sit unassisted at age 6 months, spoke first words at 12 months and walked at 13 months. At the age of 6 years, his mother noticed hearing problems and bilateral sensorineural impairment was diagnosed. Pure tone audiometry showed a nearly symmetrical loss with normal hearing up to $1 \mathrm{kHz}$ and hearing thresholds of 35 $\mathrm{dB}$ at $2 \mathrm{kHz}, 40 \mathrm{~dB}$ at $4 \mathrm{kHz}$ and $60 \mathrm{~dB}$ at $8 \mathrm{kHz}$, respectively. Speech and language had developed normally. His achievements in school and developmental testing (German version of the Developmental Test of Visual Perception) were ahead of age. The boy was fitted with hearing aids and his problems to understand at home and in school ceased. At the age of $71 / 2$ years, height was 120.3 $\mathrm{cm}$ (25th percentile) and weight $24.5 \mathrm{~kg}$ (25-50th percentile). Clinical investigation disclosed no abnormalities apart from a mild hyperopia that was also present in his younger brother. Thyroid function test, electrocardiogram and kidney ultrasound were normal, as was mutation analysis of common hearing loss genes including GJB2 and GJB6. 


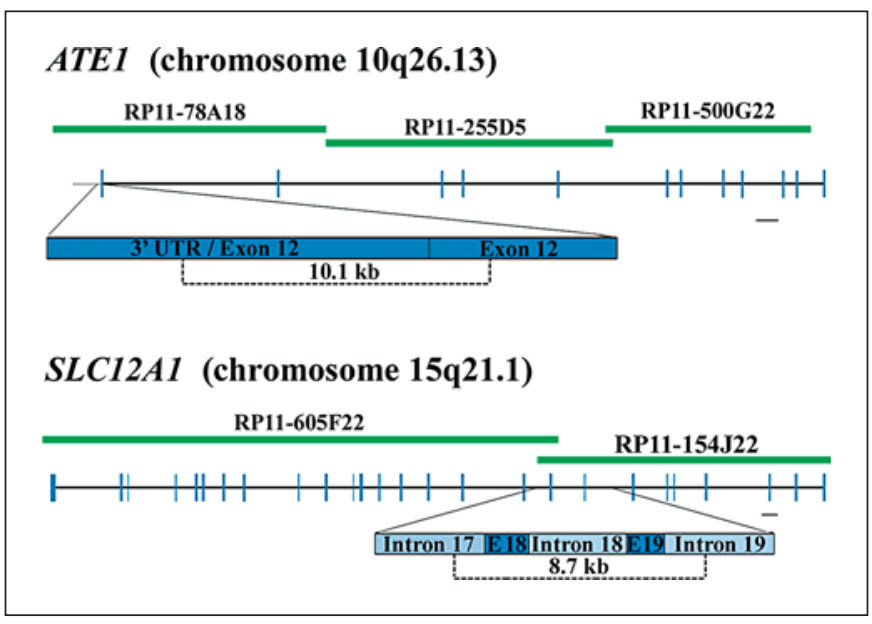

Fig. 2. Graphic illustrations showing a translocation-mediated disruption of the ATE1 and SLC12A1 genes. The upper part shows a BAC (green) contig covering the ATE1 breakpoint region on chromosome 10. Bars represent $20 \mathrm{~kb}$. An expanded representation of the breakpoint including exon 12 with the end of the coding region and the $3^{\prime}$ UTR region is shown in dark blue, and comprises a 10.1$\mathrm{kb}$ interval. The bottom part shows a BAC contig covering the SLC12A1 breakpoint on chromosome 15. An expanded representation of the breakpoint spanning introns 17-19 and exons 18 and 19 in light blue and dark blue, respectively, comprise an 8.7-kb interval.

\section{Chromosome Banding Analysis}

The patient was identified in a cytogenetic screen for disease-associated balanced chromosome rearrangements in $>300$ children with non-syndromic hearing impairment [Schneider et al., 2009]. GTG banding revealed an apparently balanced translocation between the long arms of chromosomes 10 and 15. At the cytogenetic level, the breakpoints were assigned to bands 10q26.11q26.13 and 15q21.1q21.2 (fig. 1a, b). Karyotyping of the parents and family (data not shown) revealed that the translocation had been inherited from the father and was also present in the proband's brother and paternal grandfather, all with normal hearing. The mother and the paternal grandmother had normal female karyotypes.

\section{Positional Cloning of Translocation Breakpoints}

Once the breakpoints were provisionally delineated, FISH experiments were used to refine the breakpoint location. Online supplementary table 1 lists the BAC clones that were selected with their corresponding mapping results. BAC RP11-78A18 (83 kb) hybridized to the normal chromosome 10 and both derivative chromosomes 10 and 15 (fig. 1c), as expected for a chromosome 10-breakpoint spanning clone. Similarly, we identified a break- point-spanning BAC clone, RP11-154J22 (101 kb) on chromosome 15. Based on the FISH results, the proband's karyotype was refined to $46, \mathrm{XY}, \mathrm{t}(10 ; 15)(\mathrm{q} 26.13 ; \mathrm{q} 21.1)$.

To further narrow down the breakpoint regions, we generated long-range PCR products from the breakpointspanning clones on chromosomes 10 and 15 (online suppl. table 2). The FISH mapping of individual longrange PCR products assigned breakpoints to a $10.1-\mathrm{kb}$ interval (amplicon 6 of BAC RP11-78A18) on chromosome 10 localizing to exon 12 including the end of the coding sequence and the $3^{\prime}$ UTR of the ATE 1 gene and an 8.7-kb interval (amplicon 2b of BAC154J22) on chromosome 15 between introns 17 and 19 of the SLC12A1 gene. Breakpoint intervals are illustrated in figure 2.

Consistent with the UniGene database (http://www. ncbi.nlm.nih.gov/unigene), we found that ATE1 but not SLC12A1 is expressed in blood. Although we localized the translocation breakpoints to predicted regions within 2 genes, we were unable to successfully detect fusion gene transcripts in the proband (data not shown). However, because of the $3^{\prime}-5^{\prime}$ orientation of ATE1 and the $5^{\prime}-3^{\prime}$ orientation of SLC12A1 in the derivative chromosomes, the possibility of an ATE1-SLC12A1 or SLC12A1-ATE1 fusion transcript is unlikely due to the opposing transcriptional directions.

In addition to direct gene disruption, the translocation could indirectly affect the regulation of genes within a flanking one megabase region of ATE1 and SLC12A1. There are several interesting candidate genes in these regions that, while not associated with a deafness phenotype, demonstrate expressed sequence tags in the human ear (online suppl. table 4).

\section{Mutation Analyses}

According to DECIPHER version 5.1 (http://decipher. sanger.ac.uk), both ATE1 and SLC12A1 are not thought to affect the phenotype by way of haploinsufficiency. In order to test whether gene disruption by translocation unmasks a recessive mutation in the second allele of our proband, we sequenced all 12 coding exons of ATE1 (MIM 604103) and all 26 coding exons of SLC12A1 (MIM 600839 ) and did not detect any pathogenic mutation. $\mathrm{He}$ was homozygous for 2 synonymous SNPs c.303A $>\mathrm{G}$ (rs10749435) and c.1236A $>\mathrm{G}$ (rs4237536) in ATE1 and heterozygous for one non-synonymous benign SNP c.2873T>C, p.Val958Ala (rs1552311) in SLC12A1.

In addition, we performed a mutation screening in 180 GJB2 mutation-negative children with non-syndromic hearing impairment. Fifteen to $20 \%$ had mild, the remaining cases moderate to profound hearing impairment. Al-
Vona et al. 
Table 1. Results for ATE1 and SLC12A1 mutation screening in 180 patients

\begin{tabular}{|c|c|c|c|c|c|c|}
\hline \multirow[t]{7}{*}{ ATE1 (exons 1-12) } & 1 & c. $9 \mathrm{C}>\mathrm{T}$ & p.Phe3Phe & rs79570924 & & \\
\hline & 1 & c. $88 \mathrm{~T}>\mathrm{G}$ & p.Ser30Ala & & benign $(0.000)$ & tolerated $(0.83)$ \\
\hline & 3 & c. $198 \mathrm{C}>\mathrm{T}$ & p.Val66Val & & & \\
\hline & 5 & c. $499 \mathrm{C}>\mathrm{T}$ & p.Leu167Phe & rs148095496 & benign $(0.000)$ & tolerated $(0.09)$ \\
\hline & $\underline{9}$ & c. $1125 \mathrm{G}>\mathrm{A}$ & p.Ser375Ser & rs139300996 & & \\
\hline & 9 & c. $1140 \mathrm{C}>\mathrm{T}$ & p.Gly380Gly & rs150860078 & & \\
\hline & 10 & c. $1208 \mathrm{~A}>\mathrm{G}$ & p.Tyr403Cys & rs148135505 & $\begin{array}{l}\text { probably damaging } \\
(1.000)\end{array}$ & $\begin{array}{l}\text { deleterious } \\
(0.00)\end{array}$ \\
\hline \multirow[t]{5}{*}{ SLC12A1 (coding exons 2-27) } & 7 & c. $828 \mathrm{G}>\mathrm{A}$ & p.Val276Val & rs3825960 & & \\
\hline & 13 & c.1539C>T & p.Val513Val & & & \\
\hline & 14 & c. $1614 \mathrm{~T}>\mathrm{C}$ & p.Tyr538Tyr & rs6493311 & & \\
\hline & 18 & c. $2067 \mathrm{G}>\mathrm{A}$ & p.Gly689Gly & rs35783293 & & \\
\hline & 24 & c. $2873 \mathrm{~T}>\mathrm{C}$ & p.Val958Ala & rs 1552311 & benign $(0.000)$ & tolerated $(0.77)$ \\
\hline
\end{tabular}

together, the ATE1 gene sequencing identified 7 synonymous, 2 benign non-synonymous and 1 probably damaging or deleterious base change (table 1). The potentially pathogenic c.1208A $>\mathrm{G}$ (p.Tyr403Cys) mutation was present in a heterozygous state in a single individual with severe hearing impairment in the lower frequencies that improves to normal hearing at higher frequencies (particularly at 6 and $8 \mathrm{kHz}$ ). She also suffers from recurrent bilateral tinnitus for at least 10 years. This mutation occurs in a highly conserved nucleotide and amino acid, up to Baker's yeast (considering 12 species) and has a large physicochemical difference existing between Tyr and Cys with a Grantham distance of 194 (Alamut version 2.0). This variation is also entered in dbSNP (http://www.ncbi.nlm.nih.gov/SNP) as rs 148135505 , with a MAF of 0.001 (1000 Genomes) and is in the Exome Variant Server database (http://evs.gs.washington.edu) with a similar MAF of 0.002 in the European population. The SLC12A1 sequencing detected 4 synonymous and 1 non-synonymous variant (table 1 ).

Translocation-Mediated Disruption of ATE1 and SLC12A1
Targeted Next Generation Sequencing of Deafness

Genes and SNP Array Analysis

Because the translocation was segregating in both affected and unaffected family members and we did not find evidence for mutations in ATE1 and SLC12A1 underlying hearing impairment, we used targeted deafness gene enrichment sequencing (Otogenetics) to screen for mutations in 130 known deafness genes. The analysis strategy we employed disclosed only apparently nonpathogenic variants. Notably, one non-synonymous heterozygous change, c.1985G >A, p.Gly662Glu, in myosin IA (MYO1A, MIM 601478), a major contributor to autosomal dominant hearing loss (DFNA48) [Donaudy et al., 2003], was reported as possibly pathogenic by UniProt through the Alamut mutation report. However, because this variant is in a position of the MYO1A motor domain that is not evolutionarily conserved and also listed as a SNP (rs33962952), it is unlikely to cause hearing impairment. The fact that this SNP was found in the normally 

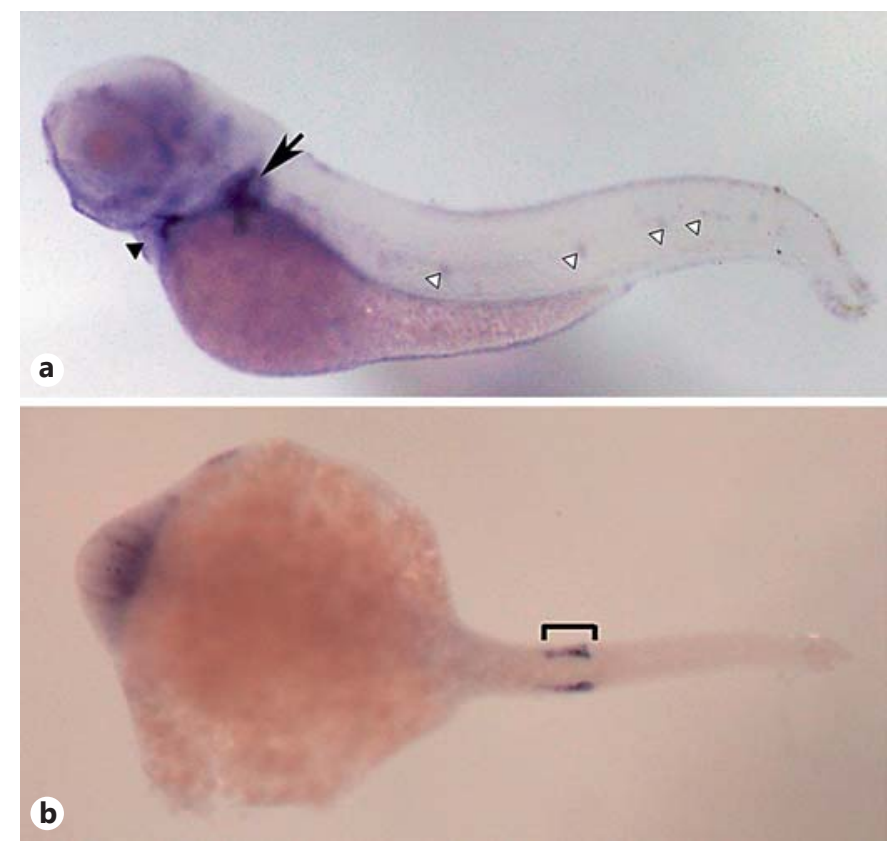

Fig. 3. Whole-mount in situ hybridization of wild-type zebrafish embryos shows the expression pattern of ate 1 and slc12a1 during embryogenesis. a 91-hpf lateral view depicting strong ate 1 expression in the fin bud (arrow) and heart (filled arrowhead) with minor expression in neuromasts (open arrowheads). $\mathbf{b}$ A polar view of the 15-16-hpf somite stage with slc12al expression in the distal early pronephros (bracket).

hearing father and paternal grandfather of our proband also argues against a dominant causative mutation, although the formal possibility of reduced penetrance in unaffected family members cannot be excluded.

In some cases of apparently balanced chromosome rearrangements, microdeletions and/or duplications in the vicinity of the breakpoint regions [Yue et al., 2005] or elsewhere in the genome [Baptista et al., 2008] were found to be disease-causing. The SNP array analysis did not reveal any cryptic gain or loss of chromosome material in the proband (data not shown). There was no detectable difference in the breakpoint regions between affected and unaffected tranlocation carriers. The proband displayed a $4-\mathrm{Mb}$ copy neutral loss of heterozygosity on chromosome 17 (50,329,974-54,383,237 bp; NCBI 37/hg19); however, this region does not contain any strong deafness candidate genes. Interestingly, when investigating known copy number variation in the breakpoint intervals in the database of genomic variants [Zhang et al., 2006], ATE1 lacks genomic variation and $S L C 12 A 1$ has only one indel reported in 270 HapMap controls.

\section{Expression of ate1 and slc12a1 during Zebrafish Development}

According to UniGene, neither ATE1 nor SLC12A1 appears to be expressed in the human ear. To evaluate the possible functional role of ATE1 and SLC12A1 during ear development, the expression patterns were investigated in zebrafish embryos using ate1 and slc12al cDNA probes. Major expression of ate 1 was marked at $91 \mathrm{hpf}$ in the heart and fin bud, with minor expression in the neuromasts (fig. 3a). Prominent expression of slc12a1 was observed in the distal early pronephros in all stages beginning with the somite stage at 15-16 hpf, at $30 \mathrm{hpf}$ and in $96 \mathrm{hpf}$ (fig. 3b). As expected, no signal was visualized with the sense riboprobes.

\section{Discussion}

The proband inherited a balanced translocation $\mathrm{t}(10$; 15) from his phenotypically normal father; thus, it is unlikely that the translocation alone is by itself the cause of deafness. However, interplaying with other genetic and/ or environmental factors, it could contribute to the events leading to hearing loss. Upon the identification of the 2 disrupted genes in the breakpoint regions (ATE1 in $10 \mathrm{q} 26.13$ and $S L C 12 A 1$ in $15 \mathrm{q} 21.2$ ), we began a candidate gene approach involving bidirectional sequencing of all exons of each of the disrupted genes in the proband as well as a pool of 180 hearing impaired individuals.

To date, there are no disease-causing mutations or phenotypes associated with ATE1 in humans that have been cataloged in databases such as HGMD (http://www. biobase-international.com/product/hgmd) and SwissVar (http://swissvar.expasy.org). Evidence from Ate1 $1^{-/}$ knockout mice suggests that this gene is important for cardiovascular development [Kwon et al., 2002]. We show strong expression of the zebrafish ortholog in the developing heart (and fin bud). To the best of our knowledge, neither the proband nor affected family members with the described translocation have heart defects. An interesting point for discussion is that there was weak expression of ate 1 observed in the neuromasts of zebrafish. Neuromasts are, in morphology and functionality, similar to hair cells of the ear. Consistent with the in silico prediction (DECIPHER) that ATE1 is not sensitive to haploinsufficiency, heterozygous disruption of ATE1 alone can be tolerated. Posttranslational arginylation by ATE1 is an important biological regulator of protein properties and function. The tRNA-dependent addition of Arg onto proteins is essential for mammalian embryo- 
genesis and tissue morphogenesis [Saha and Kashina, 2011]. In this light, it is tempting to speculate that arginylation of a potential ATE1-target protein, which is essential for normal hearing development, is altered by a second mutation that is present in the genome of the proband, but not in his healthy brother and father. Arginylation of hearing loss genes is not well investigated. However, differential arginylation of actin isoforms is well known [Zhang et al., 2010] and both beta and gamma actins have specific roles for auditory hair cells [Perrin et al., 2010].

In our cohort of hearing-impaired individuals, we found one potentially pathogenic mutation affecting a highly conserved nucleotide and amino acid (c.1208A $>\mathrm{G}$, p.Tyr403Cys) in the ATE1 gene in a heterozygous state. Together with our translocation patient, 2 of 180 studied individuals exhibit only one wild-type ATE1 allele. However, we have not assessed the pathogenicity of the identified mutations on a functional level. The Exome Variant Server database contains 2 additional probably pathogenic frameshift mutations which are not in dbSNP v135. The c.427_430del4 deletion with a MAF of 0.004 in Europeans affects the protein by inducing a premature stop codon (p.Leu143Valfs*8). The c.93_94insC insertion (p.Ser32Glnfs*72) with a MAF of 0.04 is listed with a read depth of only 8 and, therefore, a sequencing artifact cannot be excluded.

Homozygous or compound heterozygous mutations in SLC12A1 are associated with antenatal Bartter syndrome type I (MIM 601678), a life-threatening renal tubular disorder [Simon et al., 1996]. In this light, it is not unexpected that our zebrafish experiments showed strong slc12a1 expression in the pronephros. None of the translocation carriers were reported to suffer from renal problems. However, it is noteworthy that 2 of 9 Costa Rican Bartter syndrome patients with a homozygous premature stop mutation in the SLC12A1 gene exhibited sensorineural hearing loss [Kurtz et al., 1997]. Considering that the incidence of Bartter syndrome (with at least 5 different types) is approximately 1 in 800,000-1,000,000 (http:// www.orpha.net), the frequency of heterozygous SLC12A 1 mutation carriers is estimated to be approximately 1 in 1,000 . The risk of our index patient for a child with Bartter syndrome is then approximately 1 in 4,000.

In conclusion, heterozygous disruption of ATE 1 and/ or SLC12A1 gene function is benign in nature and does not cause a haploinsufficient state. Additionally, from sequencing data, the proband lacks a second mutation in either of these genes. Since we eliminated the contribution of another known deafness gene by performing sequencing of 130 genes associated with deafness, we can argue in favor of the notion that the proband suffers from a polygenic or multifactorial form of deafness. On the other hand, we cannot exclude the possibility that the translocation and hearing impairment are coincidental or that our proband has a pathological mutation in a gene that is not yet associated with hearing loss. Collectively, our study emphasizes the difficulty of finding causative mutations in patients with non-syndromic hearing loss. Such particularly challenging patients may become targets for novel techniques such as whole exome or genome sequencing.

\section{Acknowledgements}

The authors thank the proband and his family for participating in this study. We also thank Stefanie Kleinmichel and Katharina Eirich for help with sequencing analyses, Dr. Natalja Damatova for SNP array experiments and analysis, Dr. Jörg Schröder for clinical information, and Prof. Manfred Schartl for providing the zebrafish embryos. This work was supported by the German Research Foundation (HA 1374/7-2).

\section{References}

-Adzhubei IA, Schmidt S, Peshkin L, Ramensky VE, Gerasimova A, et al: A method and server for predicting damaging missense mutations. Nat Methods 7:248-249 (2010).

- Bache I, Hjorth M, Bugge M, Holstebroe S, Hilden $\mathrm{J}$, et al: Systematic re-examination of carriers of balanced reciprocal translocations: a strategy to search for candidate regions for common and complex diseases. Eur J Hum Genet 14:410-417 (2006).
Baptista J, Mercer C, Prigmore E, Gribble SM, Carter NP, et al: Breakpoint mapping and array CGH in translocations: comparison of a phenotypically normal and an abnormal cohort. Am J Hum Genet 82:927-936 (2008).

Bugge M, Bruun-Petersen G, Brøndum-Nielsen K, Friedrich U, Hansen J, et al: Disease associated balanced chromosome rearrangements: a resource for large scale genotype-phenotype delineation in man. J Med Genet 37:858-865 (2000).
Translocation-Mediated Disruption of ATE1 and SLC12A1
Mol Syndromol 2014;5:3-10 DOI: $10.1159 / 000355443$
Damatova N, Beyer V, Galetzka D, Schneider E, Napiontek U, et al: Haploinsufficiency of 16.4 $\mathrm{Mb}$ from chromosome 22pter-q11.21 in a girl with unilateral conductive hearing loss. Cytogenet Genome Res 125:241-247 (2009).

Donaudy F, Ferrara A, Esposito L, Hertzano R, Ben-David O, et al: Multiple mutations of MYO1A, a cochlear-expressed gene, in sensorineural hearing loss. Am J Hum Genet 72 : 1571-1577 (2003).

ATE1 and SLC12A1 
Friedman T, Griffith AJ: Human nonsyndromic sensorineural deafness. Annu Rev Genomics Hum Genet 4:341-402 (2003).

-Higgins AW, Alkuraya FS, Bosco AF, Brown KK, Bruns GA, et al: Characterization of apparently balanced chromosomal rearrangements from the developmental genome anatomy project. Am J Hum Genet 82:712-722 (2008).

Hook EB, Hamerton JL: The frequency of chromosome abnormalities detected in consecutive newborn studies - differences between studies - results by sex and by severity of phenotypic involvement, in Hook EB, Porter IH (eds): Population Cytogenetics - Studies in Humans, pp 63-79 (Academic Press, New York 1977)

Kleinjan DA, van Heyningen V: Long-range control of gene expression: emerging mechanisms and disruption in disease. Am J Hum Genet 76:8-32 (2005).

Kurtz CL, Karolyi L, Seyberth HW, Koch MC, Vargas R, et al: A common NKCC2 mutation in Costa Rican Bartter's syndrome patients: evidence for a founder effect. J Am Soc Nephrol 8:1706-1711 (1997).

Kwon YT, Kashina AS, Davydov IV, Hu RGH, An JA, et al: An essential role of $\mathrm{N}$-terminal arginylation in cardiovascular development. Science 297:96-99 (2002).
Morton CC, Nance WE: Newborn hearing screening - a silent revolution. N Engl J Med 354:2151-2164 (2006).

Ng PC, Henikoff S: Predicting deleterious amino acid substitutions. Genome Res 11:863-874 (2001).

-Perrin BJ, Sonnemann KJ, Ervasti JM: $\beta$-actin and $\gamma$-actin are each dispensable for auditory hair cell development but required for stereocilia maintenance. PLoS Genet 6:e1001158 (2010).

- Saha S, Kashina A: Posttranslational arginylation as a global biological regulator. Dev Biol 358: 1-8 (2011).

Schneider E, Märker T, Daser A, Frey-Mahn G, Beyer V, et al: Homozygous disruption of $P D Z D 7$ by reciprocal translocation in a consanguineous family: a new member of the Usher syndrome protein interactome causing congenital hearing impairment. Hum Mol Genet 18:655-666 (2009).

Simon DB, Karet FE, Hamdan JM, DiPietro A, Sanjad SA, Lifton RP: Bartter's syndrome, hypokalaemic alkalosis with hypercalciuria, is caused by mutations in the $\mathrm{Na}-\mathrm{K}-2 \mathrm{Cl}$ cotransporter NKCC2. Nat Genet 13:183-188 (1996).
-Warburton D: De novo balanced chromosome rearrangements and extra marker chromosomes identified at prenatal diagnostics: clinical significance and distribution of breakpoints. Am J Hum Genet 49:995-1013 (1991).

Williamson RE, Darrow KN, Michaud S, Jacobs JS, Jones MC, et al: Methylthioadenosine phosphorylase (MTAP) in hearing: gene disruption by chromosomal rearrangement in a hearing impaired individual and model organism analysis. Am J Med Genet A 143A:1630-1639 (2007)

-Winkler C, Moon RT: Zebrafish mdk2, a novel secreted midline, participates in posterior neurogenesis. Dev Biol 229:511-515 (2001).

-Yue Y, Grossmann B, Holder SE, Haaf T: De novo $\mathrm{t}(7 ; 10)(\mathrm{q} 33 ; \mathrm{q} 23)$ translocation and closely juxtaposed microdeletion in a patient with macrocephaly and developmental delay. Hum Genet 117:1-8 (2005).

Zhang F, Saha S, Shabalina SA, Kashina A: Differential arginylation of actin isoforms is regulated by coding sequence-dependent degradation. Science 329:1534-1537 (2010).

Zhang J, Feuk L, Duggan GE, Khaja R, Scherer SW: Development of bioinformatics resources for display and analysis of copy number and other structural variants in the human genome. Cytogenet Genome Res 115:205-214 (2006). 